(C) 2014 IEEE. Personal use of this material is permitted. Permission from IEEE must be obtained for all other uses, in any current or future media, including reprinting/republishing this material for advertising or promotional purposes, creating new collective works, for resale or redistribution to servers or lists, or reuse of any copyrighted component of this work in other works. 


\section{Energy-constrained paths for optimization of energy consumption in Wireless Sensor Networks}

\author{
Doan B Hoang \\ iNEXT- Centre for Innovation in IT Services and \\ Applications \\ University of Technology, Sydney \\ Australia \\ Doan.Hoang@uts.edu.au
}

\author{
Najmeh Kamyabpour \\ iNEXT-Centre for Innovation in IT Services and \\ Applications \\ University of Technology, Sydney \\ Australia \\ najmeh@it.uts.edu.au
}

\begin{abstract}
A sensor spends a large part of its energy in transmitting its data and relay its neighbours' data. The overall lifetime of a wireless sensor network depends strongly on how a sensor selects its relaying neighbours and the data path to the destination. One critical problem is that if a sensor has to support too many neighbours, its energy is exhausted rapidly and may bring down the whole network. This paper suggests algorithms for assigning weights to links between neighbours taking into account the number of neighbours who rely on them to relay traffic to the destination. In order to do so, the paper also proposes an algorithm for constructing node connectivity based on sensors position within the broadcast range of another sensor, and a shortest energy-constrained path from a sensor to the destination.
\end{abstract}

Keywords-energy minimization; energy-constrained path; energy driven architecture; node-connectivity algorithm; weight assignment algorithm; shortest path

\section{INTRODUCTION}

A wireless sensor always relies on its battery to operate. Yet with this limited energy source, the sensor has to work collaboratively with other sensors in a wireless sensor network to deliver data to a destination as dictated by the application. As a consequence, sensors and wireless sensor networks' usefulness in real-life applications are severely constrained.

The challenge is how to minimize the energy consumption of a sensor network taking into account of various constraints of the application.

It is generally expected that by reducing any energy consumption components would reduce the overall of the overall energy consumption of the whole network. Hence, current efforts in minimizing energy consumption have increased over the last few years mostly focused on some specific and separate components of energy dissipation in WSNs such as MAC protocols [1], [2], [3], routing [4], topology management [5] and data aggregation [6]. However, these components are highly integrated within a WSN and their interplay, in terms of energy consumption, cannot be taken into account because each constituent is treated independently without regard for other constituents.

In our earlier work [7], [8], we proposed two energydriven models: EDM-S to model the energy constituents of a sensor and EDM-WSN to formulate the total energy consumption of wireless senor networks.

Our EDM-S models the energy consumption of an individual sensor from a sensor-centric viewpoint: a sensor node within a WSN spends its energy on three constituents: the individual constituent (the existence of the sensor itself), the local constituent (the sensor as a member of its local community), and the global constituent (as a member of the sensor network).

Our (EDM-WSN) models the total energy consumption of all sensors within the network based on the topology, configuration, and routing schemes selected for the application.

Our results indicated that the individual constituent of a sensor consumes a necessary portion of its energy. This component can be minimized by designing the sensor based on the application and on the range of its sensing radius. The local constituent depends on how a sensor selects its neighbors (how many and which ones) in order to ensure that it can reliably send its data to the destination. This constituent also depends on the transmitting radius (a design parameter) or the distance between the sensor and its neighbours. The global constituent plays a significant part in shaping the total energy consumption of the whole sensor network. The results demonstrated that transmitting and receiving data packet demand a large portion of the sensor energy hence it is this global constituent one ought to focus one's attention in designing wireless sensor networks.

As a result, a number of challenging issues associated with this global constituents need to be investigated. These include: i) developing and evaluating self-organizing protocols that use a minimum number of neighbours for guaranteeing a reliable operation, ii) selecting the optimum distance between a sensor and its neighbours so as to achieve a balance between the required number of neighbours and the number of hops to the destination, iii) configuring and determining the optimum number of neighbours a sensor is willing to act as a relay along the data path to the destination, iv) finding the shortest energy path from a sensor to its data destination, v) adapting the overall network to minimize the total energy consumption of the whole application.

This paper focuses on this global constituent. In particular, this paper focusses on three aspects: i) algorithms for organizing and labeling sensors in such a way that the path of a sensor to the destination can be established for 
reliability, ii) algorithms for determining the weight of the link of the graph taking into account node's energy constraints, and iii) algorithms for selecting the shortest energy path from a sensor to its destination.

The remaining of the paper is organized as follows. The second section provides a brief review of our energy driven model. The third section describes the problem and the solution approach. The fourth section IV presents our proposed algorithms. The fifth section presents simulation results. The last section concludes the paper with suggestion for future work.

\section{THE ENERGY DRIVEN MODEL (EDM)}

In the EDM-S model, the individual constituent represents essentially the energy component that allows the sensor to exist and perform its sensing function, isolated from all other sensor nodes. The local constituent represents the energy component that allows the sensor to interact with its neighours and maintain a local community (and use them for relaying its data). The global constituent represents the energy component that allows the sensor to participate in the construction and maintenance of the network and to transport their data collectively to the destination.

The global energy consumption constituent is concerned with global strategies for maintaining the whole sensor network and for transporting all sensors' data to the destination, the sink. Transmitting and receiving data packets from hop to hop to the destination consumes sensor's energy and hence selecting relevant network topologies, data path and choosing efficient routing methods become major considerations. Adopting a network topology may depend on the objective of the application. Routing methods help minimize the number of relay hops to the destination which are expensive in terms of energy dissipation. Furthermore, inappropriate topology and routing may create congestion and packet loss hence increasing energy consumption of the network.

The total energy consumption of a wireless sensor network clearly depends of the intended application. Our model (EDM-WSN) identifies a number of parameters related to Individual, Local and Global constituents:

- The number of sensors in the WSN application, $N$

- The topology of the WSN and hence the number of neighbours of a sensor, $k$

- The routing scheme for transporting data to data collector and hence the number of hops, $h$, from a sensor to its sink.

EDM-WSN estimates the total energy consumption of a WSN for a duration of $\Delta \mathrm{t}$ as follows:

$$
E_{\text {Total }}(\Delta t)=\sum_{i=1}^{N} I_{\text {individuali }}+\sum_{i=1}^{N} k_{i} L_{\text {local }, i}+\sum_{i=1}^{N} h_{i} G_{\text {globali }}
$$

For the duration of $\Delta t, I_{\text {individual }}$ takes into account the amount of energy consumption of sensor $i$, its type and its sensing radius; $L_{\text {local }}$ takes into account the energy consumption of a sensor and one of its neighbours; and $G_{\text {global }}$ takes into account the energy consumption of the task of sending data over one hop.

$k_{i}$ in the above equation depends on the configuration of the sensor network (for example: hexagonal, square, circle neighborhood) and the communication protocol employed (directional or bidirectional); $k_{i}$ may be replaced by $f\left(k_{i}\right)$ or an average number of neigbours for all nodes in network.

$\mathrm{h}_{i}$ in the above equation can be simplified by taking it as the average number of hops ( $\bar{h}$ )from any sensor to the sink node.

\section{THE CONSTRAINED SHORTEST ENERGY PATH PROBLEM}

\section{A. Prolem description}

Our investigation established that (from the sensor-centric view)

$G_{\text {global }}$ is the dominant component contributing to the overall energy consumption of a wireless sensor. This component mainly represents the energy costs of sending and receiving data over a hop (a link between a sensor and a neighbour).

The last component of equation (1), $\sum_{i=1}^{N} h_{i} G_{\text {globali }}$, represents the sum of the energy required to send data from a sensor to the network sink for all $N$ sensors. $h_{i}$ is the number of hops from the sensor $i$ to the sink.

Clearly, the energy for transmitting and receiving data per hop and the number of hops to the sink are the two major components.

Per-hop transmission depends directly on the maximum distance between a sensor and its neighbours. In fact, the energy consumption is proportional to $d^{2}$ (where $d$ is the distance between the sensor and its neighbour, or the transmission radius). With large $d$, more nodes can be included as reachable neighbours and that results in fewer hops are required to reach the sink. Conversely, smaller $d$ means more hops are needed.

The number of hops required to reach the sink from a sensor clearly depends on the network topology, sensor density within the sensor field, the transmitter radius, the routing scheme, and other constraints such as realistic capability of sensor nodes as well as the traffic requirements.

The constrained shortest energy path problem. Given a sensor field that is covered by a fixed number of sensors and a fixed transmission radius (a sensor is a neighbour of another sensor if it is within the transmission radius of the other node); find a scheme for selecting a relaying neighbour in a way that the overall energy consumption of an individual sensor is minimized and also find a shortest path from a sensor to its destination. 
The ultimate aim is to lengthen the lifetime of the wireless sensor network.

\section{B) Assumptions}

- We assume one data sink in the network and its location is fixed within the sensor field.

- Sensors are able to communicate directly with peers within its transmission radius. That is each sensor can broadcast its packets to all surrounding stations within its range. Multi-hop relaying is used to communicate with nodes beyond its range

- $\quad d$ is a design factor and is not considered in this paper.

- The network operates in a time slice scale.

\section{C) Definitions}

- The time between network organization and the next reorganization is called a time slice.

- A sensor life time is the duration over which the sensor has adequate energy level to perform its sensing function and transmit the data to its neighbours.

- Overall energy is defined as the residual energy of all alive sensors in the network.

- Sensor physical level: The lowest number of hops from a given node to the sink is called the sensor physical level

- Sensor input degree and output degree: The number of communication links over which a sensor receives packets from its neighbours and relay them to the sink is called the input degree.

- $\quad$ The number of links that a sensor uses to send (or relay) data packets to the sink is called the output degree.

\section{PROPOSED ALGORITHMS}

Sensors are able to communicate directly with peers within its transmission radius. That is each sensor can broadcast its packets to all surrounding stations within its range.

\section{B. Node-Connectivity labelling algorithm}

The first thing is to create a connectivity graph of all sensors in the network. The graph describes all possible links between node depending on their position and their transmission radius. Nodes on this graph are labeled according to their level relative to the sink.

Node-Connectivity labeling algorithm: Starting from the sink node (labeled " 0 "), we proceed as follows. All sensors within the transmission radius of the sink are labeled " 1 " $\left(L_{u}\right.$ $=1$ ); all sensors within the transmission radius of level 1 sensors are labeled " 2 " $\left(L_{u}=2\right)$; all sensors within the transmission radius of level 2 sensors are labeled " 3 "; this process continues until all sensors are labeled.

Associated with each node is its degree. The node's degree is the number of reachable (within the node's transmission radius) between the node and its neighbours at a higher level than itself. Fig. 1 illustrates the algorithm for this process.

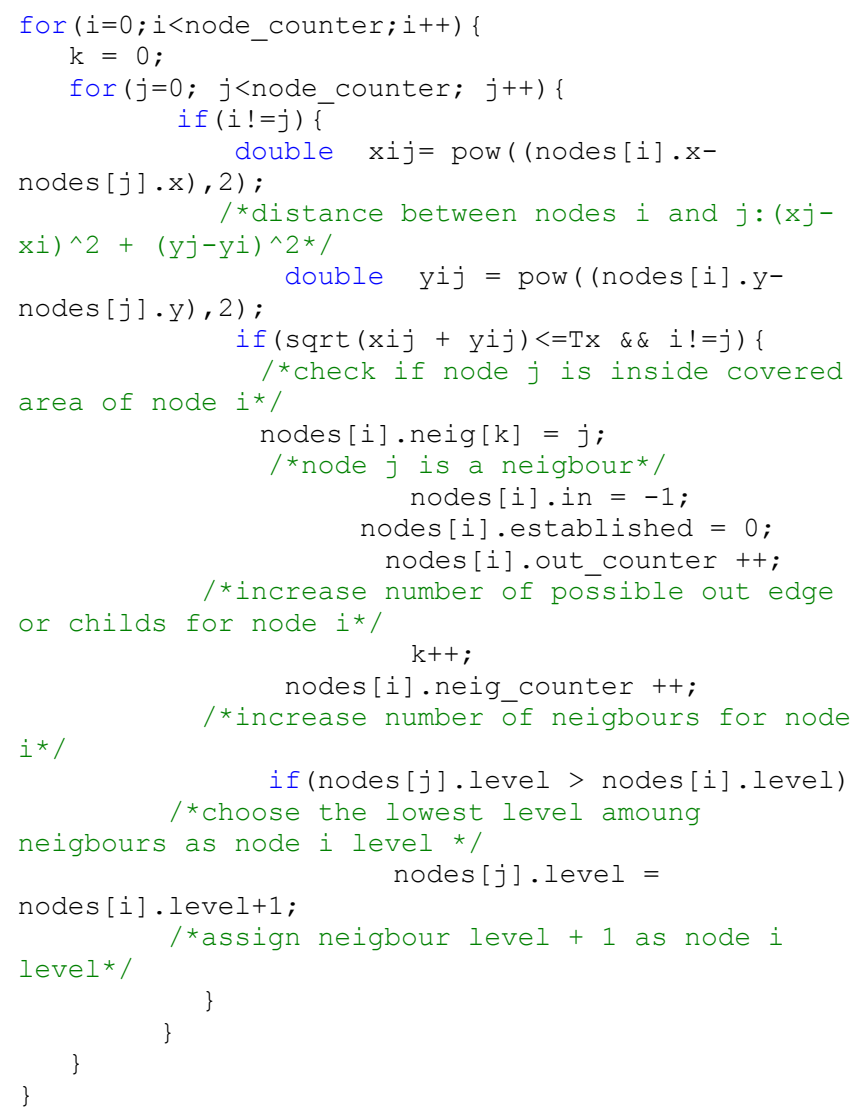

Figure 1. Node and Edge labelling algorithm.

Where $L_{u}$ is the actual node level of node $u$ (or label of node $u$ ) and $d_{u}$ is the degree of node $u$

\section{Assigning link weigh to connected links}

Once the node's level and its degree have been determined by the labeling connectivity and labeling algorithm, the weight of a link between the node and its neighbours can be assigned taking into account a number of realistic energyconstraint criteria.

A sensor, besides sending its data to the sink, it also has to play its role within the network. It has relay data packets from its neighbours to the destination sink. Clearly, if a sensor is selected by too many of its neighbours for relaying their data, its energy will be exhausted rapidly and as a consequence, it will cease to operate and the whole network may not function properly. For this reason, some constraints have to be in place.

- A node with a higher degree will present a higher cost for traffic to be carried over its link as it has to consume a larger amount of energy to relay traffic from higher number neighbours.

- The cost of reaching the sink through a neighbour at the same level is usually higher than the cost through a neighbour at the next lower level, unless the next lower level node is too busy with high degree. 
- The weigh has to be positive as it presents the cost of sending data over that link.

Determining link weight: Given the labeled graph, the weight for each link will be determined based on several constraints as discussed earlier. (2) is the measure we consider in this paper

$$
\begin{aligned}
& w(u, v)=2-\left(x \frac{L_{v}}{L_{u}+1}+y \frac{1}{d_{v}+d_{u}+1}\right) \\
& 0<x<1 ; 0<y<1 ; L_{u} \geq L_{v} ; d_{v} \geq 0 \\
& w(u, v)=\text { weigh of link from nodeu to node } v \\
& \text { along the path from } u \text { to the sink }
\end{aligned}
$$

\section{Find Shortest path from each node to the sink}

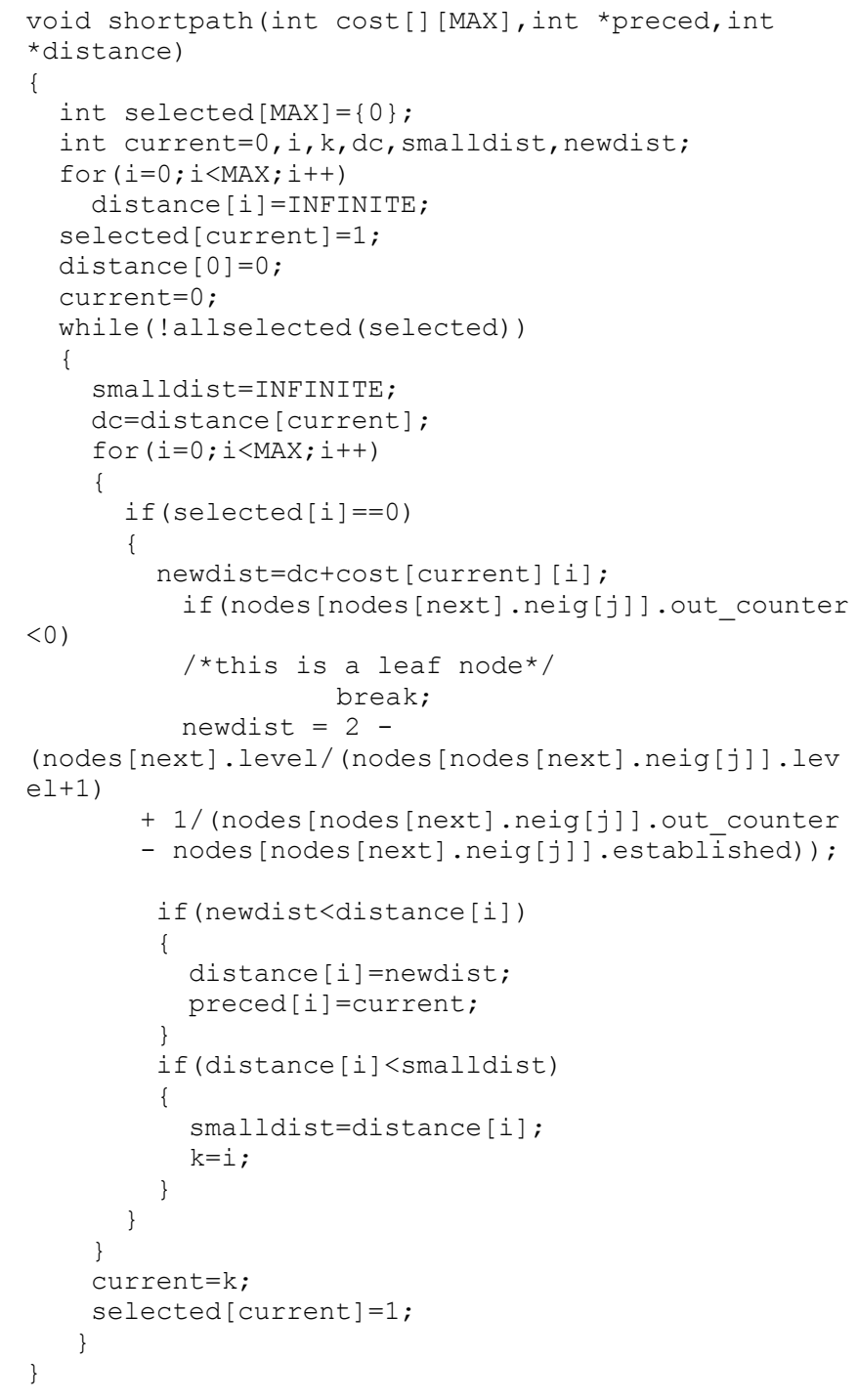

Figure 2. The shortest path algorithm given a weight graph by (2)
Once the link weights are constrained based on our measure of equation 2 and our node-connectivity labeling algorithm, shortest constrained paths from all nodes to the sink can be found by the Dijkstra's algorithm expressed above.

\section{RESULTS}

This section presents results of our proposed algorithms. Given a sensor field with 40 nodes, topologically positioned as shown in Fig. 3 with the sink is in black (disregard the labels in each node)

Fig. 3 shows the connectivity graph of the wireless sensor network after the application of the node-connectivity algorithm on over the sensor field. The first number in the circle is the sensor physical level and the second number is the input degree of the sensor.

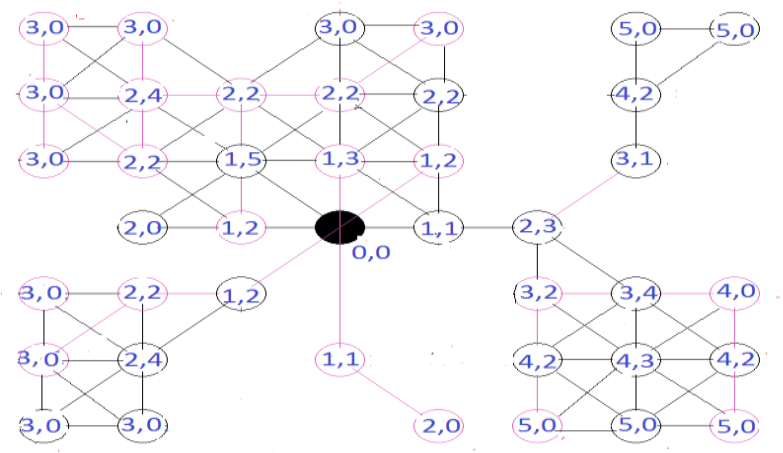

Figure 3. Node-connectivity grapth result.

Fig. 4 shows the resulting weight graph given the connectivity graph (Fig. 3) and link weight as determined by (2).

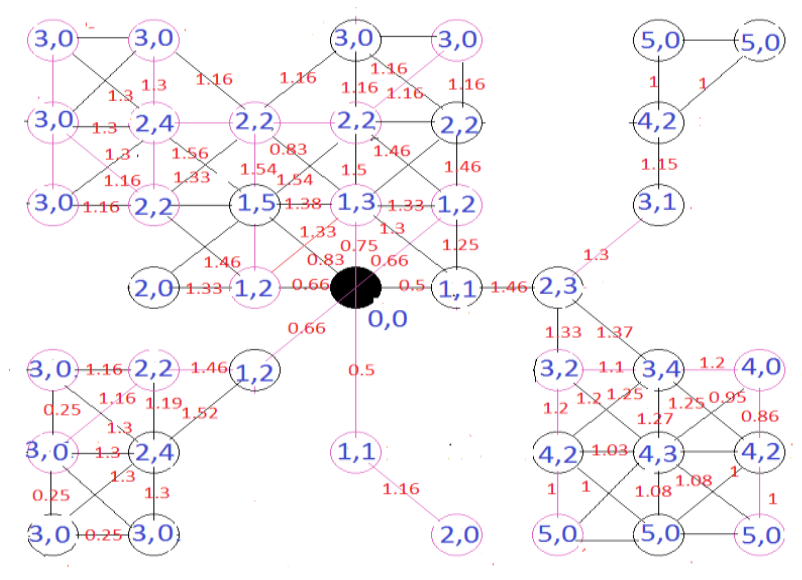

Figure 4. The resulting weight graph

Fig. 5 shows the graph of shotest paths from all nodes to the sink following the application of the shortest path algorithm. It should be noted that this paper only consider a simplest 
case where it does not takes ino account the amount of traffic generated by a node.

It should also be noted that there are other ways one can assign link weights depending on the constraints of the application. In other words, this paper presents an opportunity for considering many other important issues in optimizing the energy consumption of a wireless sensor network.

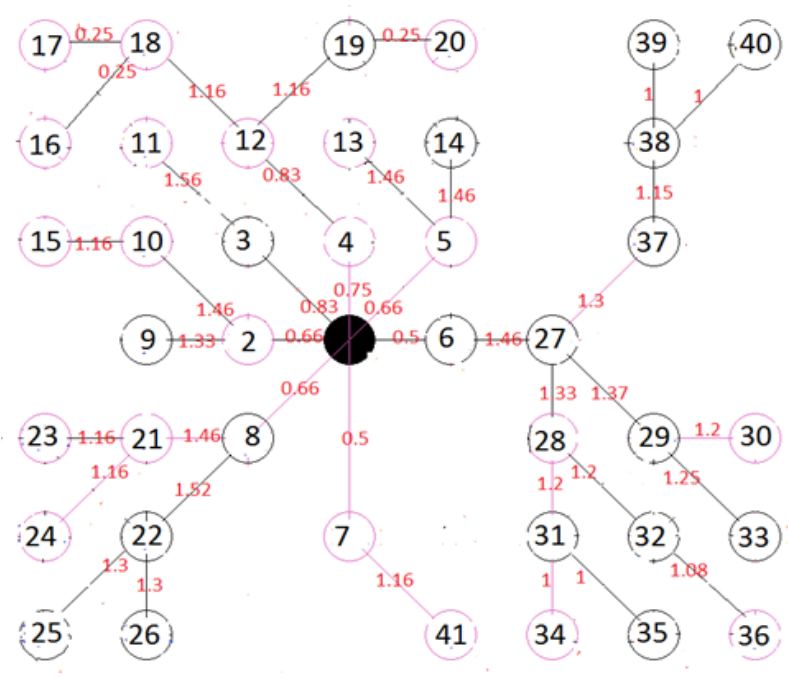

Figure 5. The constrained shortest path graph as a result of the connectivity and weight-constrained algorithms.

\section{CONCLUSION}

Based on the results of our earlier study that transporting sensed data from a sensor node to the sink in a wireless sensor network is the dominant energy consumption constituent; this paper proposed a scheme for minimizing this energy component. In particular, we proposed 1) an algorithm for creating a node-connectivity graph of the network taking into account the position of the sensors and the transmission reach of individual sensors; ii) an algorithm for assigning the link weighs between sensors based on sensor's level relative to the sink and the number of its neighbours; iii) an algorithm for finding the shortest energyconstraint path from a sensor to its sink. Many issues are open up for investigation and these will be explored in our future research. These include: i) developing and evaluating self-organizing protocols that use a minimum number of neighbours for guaranteeing a reliable operation, ii) selecting the optimum distance between a sensor and its neighbours so as to achieve a balance between the required number of neighbours and the number of hops to the destination, iii) configuring and determining the optimum number of neighbours a sensor is willing to act as a relay along the data path to the destination, iv) adapting the overall network to minimize the total energy consumption of the whole application.

\section{REFERENCES}

[1] Halkes, G.P., T. Dam, and K.G. Langendoen, Comparing energy-saving MAC protocols for wireless sensor networks. Mobile Networks and Applications Journal of Kluwer Academic, 2005. 10: p. 783-791.

[2] [2] Ye, W., J. Heidemann, and D. Estrin. An EnergyEfficient MAC protocol for Wireless Sensor Networks in IEEE Proceedings of the Infocom. 2002.

[3] Heinzelman, W.R., A. Chandrakasan, and H. Balakrishnan, Energy-efficient communication protocol for wireless microsensor networks, in 33rd Annual Hawaii International Conference on System Sciences. 2000. p. 10-20.

[4] Alzoubi, K.M., P. Wan, and O. Frieder, Distributed Heuristics for Connected Dominating Sets in Wireless Ad Hoc Networks. Journal of Communications and Networks, 2002. 4: p. 22-29.

[5] Xu, Y., et al., Topology Control Protocols to Conserve Energy in Wireless Ad Hoc Networks. 2003, Center for Embedded Networked Computing: California.

[6] [Ozdemir, S. and Y. Xiao, Secure data aggregation in wireless sensor networks: A comprehensive overview. Elsevier Journal on Computer Networks, Article in Press, 2009.

[7] Doan. B. Hoang, and N., Kamyabpour, "An Energy Driven Architecture for Wireless Sensor Networks," in 13th International Conference on Parallel and Distributed Computing, Applications and Technologies, 2012.

[8] Kamyabpour, N., and Doan. B. Hoang, "A Task Based Sensor-Centric Model for Overall Energy Consumption," 2011 in the 12th International Conference on Parallel and Distributed Computing, Applications and Technologies, 2011. 